\title{
A PROSPECTIVE STUDY ON LAPAROSCOPIC AND HISTOPATHOLOGIC DIAGNOSIS OF ENDOMETRIOSIS
}

\author{
Shilpi Mukherjee ${ }^{1}$, Sudipta Paul2, Shelly Seth³, Somajita Chakraborty 4 \\ ${ }^{1}$ Assistant Professor, Department of Obstetrics \& Gynaecology, College of Medicine and Sagore Dutta Hospital, Kolkata. \\ 2 Medical Officer, Udaynarayanpur State General Hospital, Howrah. \\ ${ }^{3}$ Assistant Professor, Department of Obstetrics \& Gynaecology, College of Medicine and Sagore Dutta Hospital, Kolkata. \\ ${ }_{4}^{4}$ Associate Professor, Department of Obstetrics \& Gynaecology, Medical College, Kolkata.
}

\section{ABSTRACT}

\section{BACKGROUND}

Endometriosis is a common benign gynaecological disorder. The true prevalence is difficult to quantify as women with disease are often asymptomatic and imaging modalities have low sensitivities for diagnosis. The primary method of diagnosis is Laparoscopy with or without biopsy for histologic diagnosis. Strict histologic criteria will confirm the surgical diagnosis in ap proximately $50-65 \%$ of cases.

\section{DESIGN, SETTING AND METHODS}

This is a prospective observational institution based study. A total of 50 patients undergoing laparoscopy were included in the study in accordance with the inclusion and exclusion criteria. The results were tabulated for comparison and standard statistical software were used for data presentation.

\section{RESULTS}

In this study, we observed that laparoscopy although adequately sensitive to diagnose endometriosis lacks the specificity except in the case of ovarian endometriosis. So laparoscopy without histological confirmation may give rise to false positive results, which may lead to overtreatment of patients. Due to the high negative predictive value of laparoscopy, absence of lesion anywhere on laparoscopy can virtually exclude the diagnosis of endometriosis, saving both time and expertise.

\section{CONCLUSION}

We conclude that laparoscopy should always be combined with histopathology for accurate diagnosis in presence of any suggestive or doubtful lesion, but is not mandatory in absence of lesion anywhere in the pelvis. The laparoscopic staging of endometriosis has a positive correlation with histologic diagnosis of endometriosis.

\section{KEYWORDS}

Endometriosis, Laparoscopy, Ovarian, Gynaecology.

HOW TO CITE THIS ARTICLE: Mukherjee S, Paul S, Seth S, et al. A prospective study on laparoscopic and histopathologic diagnosis of endometriosis. J. Evolution Med. Dent. Sci. 2016;5(62):4369-4373, DOI: 10.14260/jemds/2016/997

\section{INTRODUCTION}

Endometriosis is the third leading cause of gynaecological admissions. It is a progressive disease which is both physically and mentally debilitating and the usual time interval between the onset of symptoms and confirmed diagnosis may average 6 years or more.[1] The primary method of diagnosis is laparoscopy with or without biopsy for histologic diagnosis.[2] Using this standard, investigators have reported the annual incidence of surgically diagnosed endometriosis to be 1.6 cases per 1,000 women aged between 15 and 49 years.

However, laparoscopy is a costly procedure with substantial anaesthetic and surgical risks including potential for visceral and vascular injury. Accurate diagnosis by laparoscopy is, therefore, essential for optimally targeting women to undergo this procedure. This will reduce unnecessary operative morbidity and efficiently use health service resources.

Financial or Other, Competing Interest: None.

Submission 25-06-2016, Peer Review 20-07-2016,

Acceptance 26-07-2016, Published 03-08-2016.

Corresponding Author:

Dr. Shilpi Mukherjee,

Flat No.-D/8, North Spring Apartments 8,

M. M. Feeder Road, P. O. - Ariadaha,

Kolkata-700057, West Bengal, India.

E-mail: dr.shilpimukherjee@gmail.com

DOI: 10.14260/jemds/2016/997
Strict histologic criteria will confirm the surgical diagnosis of endometriosis in approximately $50-65 \%$ of cases.[3] Various studies have also shown that the biopsy specimens from normal-appearing peritoneum can provide histologic confirmation of endometriosis in $0 \%$ to $13 \%$ of cases.[4]

Thus, it is not clear how accurate visualization of abnormal peritoneal lesions is in terms of the diagnosis and staging of endometriosis. In this setting, our study tries to find the correlation between the laparoscopic and histopathologic diagnosis of endometriosis.

\section{AIMS AND OBJECTIVES}

The purpose of this study is to evaluate the role of laparoscopy in the diagnosis of endometriosis, i.e. its sensitivity, specificity, Positive Predictive Value (PPV), Negative Predictive Value (NPV) and to compare its accuracy in relation to the histopathological diagnosis.

\section{MATERIALS AND METHODS}

This is a prospective observational institution-based study conducted at the Department of Obstetrics and Gynaecology, Medical College and Hospital, Kolkata, India. The patients were recruited from January 2012 to June 2013. 
Study was conducted after approval by Institutional Ethics Committee of Medical College, Kolkata. Informed written consent was taken from each subject before recruitment.

\section{Inclusion Criteria}

All patients undergoing laparoscopy under a particular unit with chronic pelvic pain (Defined as non-cyclic pelvic pain in the same location for a minimum of 6 months, which was unrelated to menses and/or sexual intercourse) and/or progressive dysmenorrhoea (Defined as cyclical pain during menstruation affecting daily activities with subjective increase over last 6 months) and/or infertility (Defined as one year of unprotected intercourse without pregnancy) or laparoscopic cystectomy. Patients with laparoscopic evidence of peritoneal endometriosis and/or chocolate cyst were also included.

\section{Exclusion Criteria}

Patients without laparoscopic evidence of pelvic endometriosis and those who were taking or had taken treatment for endometriosis within last one year were excluded from the study, because this may affect the visual appearance of endometriosis at laparoscopy.

\section{Sample Size Calculation}

The sample size was calculated by using the formula and values $\mathrm{n}=1.96^{2} \mathrm{xp}(1-\mathrm{p}) / \mathrm{X}^{2}$ where $\mathrm{X}=\mathrm{C} . \mathrm{I} .=0.05$ and $\mathrm{p}=$ sensitivity $=97 \%$. Taking the reference from an earlier study and considering $10 \%$ drop-out, the sample size of 50 was obtained for our study.

\section{Statistical Analysis}

Analysis of the statistical data was done by using Microsoft Office Excel 2007 and MedCalc version 12.5. Differences were considered statistically significant when $p$-value was less than 0.05 . Two-tailed test was conducted and 0.05 was taken as level of significance, where the Null Hypothesis has to be rejected. P-value for trend was also determined and interpreted in the same manner. Correlation between variables were determined by Kappa value $(\kappa)$, by which the degree of agreement between two raters was estimated.

\section{Data Collection at Recruitment}

Detailed history taking and clinical examination along with sonological evaluation of lower abdomen and pelvis was performed. Each consecutive patient fulfilling the inclusion and exclusion criteria was recruited for the study till the desired sample size was achieved.

\section{Laparoscopic and Histopathological Study}

All recruited patients underwent laparoscopy under general anaesthesia, during which peritoneal lesions suspicious for endometriosis were excised. In patients with endometriotic lesion elsewhere in the pelvis, areas of normal-appearing pelvic peritoneum were routinely sampled with multiple, sitespecific biopsies wherever accessible. All excised lesions were sent for pathologic diagnosis. The stage of the disease was assigned using the standards described in the revised American Fertility Society Classification System. [5]

One of our hospital pathologist who was blinded to the type of lesion (If any) reviewed haematoxylin and eosin stained slides from formalin-fixed, paraffin-embedded specimens for evidence of endometriosis. If no endometriosis was seen on the initial slide, the examination was repeated using samples from three different levels of the paraffin. Both endometrial glands and stroma were present for the lesion to be labelled as containing endometriosis.

\section{RESULTS}

From the 50 recruited patients undergoing laparoscopy, we had biopsy of total 227 sites (190 peritoneal and 37 adnexal mass) of which 139 (115 peritoneal and 24 adnexal mass) had laparoscopic evidence of endometriosis, but only 74 ( 54 peritoneal and 20 adnexal) had a positive histology. All the results were tabulated and compared.

Table 1, compares the laparoscopic and histopathologic findings in different sites. It is evident that for individual site of lesion, laparoscopic visualisation of endometriosis has fair-tomoderate agreement for histologically proven endometriosis for that particular site. Laparoscopic visualisation of chocolate cyst has very strong agreement for histopathologically proven endometrioma.

Table 2, compares the sensitivity and specificity of laparoscopically suggestive endometriotic lesion for confirmation of endometriosis at that lesion - site. It is evident that for a particular site the laparoscopy is more than $90 \%$ sensitive and has more than $90 \%$ negative predictive value for endometriosis at that site, but its specificity for endometriosis at that site varies from $42.86 \%$ to $66.67 \%$. For different peritoneal sites, the overall specificity for any peritoneal lesion for endometriosis at that site is $52.59 \%$ only and only chocolate cyst has $76.47 \%$ specificity for ovarian endometriosis. Among peritoneal sites, uterosacral ligaments showed a higher specificity compared to other sites.

Table 3, estimates the sensitivity and specificity of laparoscopically suggestive lesion for histological diagnosis of endometriosis in a patient. The sensitivity of peritoneal lesion varies from $54.55 \%$ to $75 \%$ and specificity varies from $33.33 \%$ to $54.55 \%$ depending on the site. The sensitivity and specificity of chocolate cyst for diagnosis of endometriosis is $83.33 \%$ and $69.23 \%$ respectively.

By definition histopathology has $100 \%$ specificity and $100 \%$ positive predictive value for endometriosis. But the Table 4 shows that in a patient histopathology from an individual peritoneal site has sensitivity within $41.38 \%$ to $52 \%$ for diagnosis of endometriosis. Only in presence of endometrioma, the sensitivity increases to $83.33 \%$ for diagnosis of endometriosis. So a random negative biopsy from any of the peritoneal site cannot rule out possibility of endometriosis in a patient.

We further compared the laparoscopic staging with histopathological diagnosis of endometriosis (Table 5), and found that there is no significant difference ( $p$ value $=0.2251$ ) in different stage of the disease in terms of frequency of presence or absence of histologically proven endometriosis. However, we also observed that in presence of endometriosis on histopathology there was an increasing trend ( $p$ value for trend $=0.0478$ ) with increasing stage of endometriosis.

Among the 60 sites from where biopsy could not be taken 48 were inaccessible to laparoscopy, and 12 had laparoscopic evidence of endometriosis (Table 6). It is evident that none of the laparoscopically stage 1 and stage 2 disease had any inaccessible peritoneal biopsy site, whereas in stage 3 and stage 4 patients $10(11.77 \%)$ and 50 (58.83\%) sites respectively were inaccessible to biopsy and the uterosacral ligaments were inaccessible in more than $76 \%$ cases in stage 4 disease. 


\begin{tabular}{|c|c|c|c|c|c|}
\hline Site & $\begin{array}{l}\text { Total No. } \\
\text { of Biopsy }\end{array}$ & $\begin{array}{l}\text { No. of Endometriotic } \\
\text { Site by Laparoscopy }\end{array}$ & $\begin{array}{c}\text { No. of Endometriotic Site } \\
\text { by Histopathology }\end{array}$ & $\begin{array}{l}\text { No. of False Negative } \\
\text { Site by Laparoscopy }\end{array}$ & $\begin{array}{c}\text { Kappa } \\
(\kappa)\end{array}$ \\
\hline $\begin{array}{c}\text { Right } \\
\text { Uterosacral } \\
\end{array}$ & 32 & $18(56.2 \%)$ & $9(28.1 \%)$ & $x_{2}=0$ & 0.467 \\
\hline $\begin{array}{c}\text { Left } \\
\text { Uterosacral }\end{array}$ & 33 & 17 (51.5\%) & $9(27.3 \%)$ & 0 & 0.522 \\
\hline $\begin{array}{l}\text { Pouch of } \\
\text { Douglas }\end{array}$ & 43 & 27 (62.8\%) & $12(27.9 \%)$ & 1 & 0.290 \\
\hline $\begin{array}{l}\text { Right Ovarian } \\
\text { Fossa }\end{array}$ & 41 & $28(68.3 \%)$ & $13(31.7 \%)$ & 1 & 0.269 \\
\hline $\begin{array}{l}\text { Left Ovarian } \\
\text { Fossa }\end{array}$ & 41 & $25(61 \%)$ & $11(26.83 \%)$ & 1 & 0.292 \\
\hline $\begin{array}{c}\text { Total } \\
\text { Peritoneal Site }\end{array}$ & 190 & $115(60.53 \%)$ & $54(28.4 \%)$ & 3 & 0.351 \\
\hline Adnexal Mass & 37 & $24(64.9 \%)$ & $20(54.1 \%)$ & 0 & 0.778 \\
\hline Total & 227 & $139(61.23 \%)$ & $74(32.6 \%)$ & 3 & 0.418 \\
\hline
\end{tabular}

\begin{tabular}{|c|c|c|c|c|c|}
\hline Site & Sensitivity & Specificity & PPV & NPV & Prevalence \\
\hline Right Uterosacral & $100 \%$ & $60.87 \%$ & $50 \%$ & $100 \%$ & $28.12 \%$ \\
\hline Left Uterosacral & $100 \%$ & $66.67 \%$ & $52.94 \%$ & $100 \%$ & $27.27 \%$ \\
\hline Pouch of Douglas & $91.67 \%$ & $48.39 \%$ & $40.74 \%$ & $93.75 \%$ & $27.91 \%$ \\
\hline Right Ovarian Fossa & $92.31 \%$ & $42.86 \%$ & $42.86 \%$ & $92.31 \%$ & $31.71 \%$ \\
\hline Left Ovarian Fossa & $90.91 \%$ & $50 \%$ & $40 \%$ & $93.75 \%$ & $26.83 \%$ \\
\hline All Peritoneal & $94.44 \%$ & $52.59 \%$ & $44.35 \%$ & $95.95 \%$ & $28.57 \%$ \\
\hline Adnexal Mass & $100 \%$ & $76.47 \%$ & $83.33 \%$ & $100 \%$ & $54.05 \%$ \\
\hline All Sites & $95.95 \%$ & $55.26 \%$ & $51.08 \%$ & $96.55 \%$ & $32.74 \%$ \\
\hline Tab & $\begin{array}{l}\text { ity and Sp } \\
\text { for Confirr }\end{array}$ & $\begin{array}{l}\text { Laparoscop } \\
\text { Endometrios }\end{array}$ & $\begin{array}{l}\text { Iggestive } \\
\text { t Lesion }\end{array}$ & metriotic & \\
\hline
\end{tabular}

\begin{tabular}{|c|c|c|c|c|}
\hline Site of Lesion & Sensitivity & Specificity & PPV & NPV \\
\hline Right Uterosacral & $61.90 \%$ & $54.55 \%$ & $72.22 \%$ & $42.86 \%$ \\
\hline Left Uterosacral & $54.55 \%$ & $54.55 \%$ & $70.59 \%$ & $37.50 \%$ \\
\hline Pouch of Douglas & $65.52 \%$ & $42.86 \%$ & $70.37 \%$ & $37.50 \%$ \\
\hline Right Ovarian Fossa & $75 \%$ & $33.33 \%$ & $63.64 \%$ & $46.15 \%$ \\
\hline Left Ovarian Fossa & $67.74 \%$ & $35.29 \%$ & $65.62 \%$ & $37.50 \%$ \\
\hline Chocolate Cyst & $83.33 \%$ & $69.23 \%$ & $83.33 \%$ & $69.23 \%$ \\
\hline \multicolumn{7}{|c|}{ Table 3: Sensitivity and Specificity of Laparoscopic Evidence of Endometriotic } \\
Lesion for Confirmation of Endometriosis in a Patient \\
\hline
\end{tabular}

\begin{tabular}{|c|c|c|c|c|}
\hline Site of Lesion & Kappa (k) & Sensitivity & NPV & Prevalence \\
\hline Right Uterosacral & 0.340 & $42.86 \%$ & $47.83 \%$ & $65.62 \%$ \\
\hline Left Uterosacral & 0.316 & $40.91 \%$ & $45.83 \%$ & $66.67 \%$ \\
\hline Pouch of Douglas & 0.315 & $41.38 \%$ & $45.16 \%$ & $67.44 \%$ \\
\hline Right Ovarian Fossa & 0.458 & $52 \%$ & $57.14 \%$ & $60.98 \%$ \\
\hline Left Ovarian Fossa & 0.349 & $42.31 \%$ & $50 \%$ & $63.41 \%$ \\
\hline Endometrioma & 0.778 & $83.33 \%$ & $76.47 \%$ & $64.86 \%$ \\
\hline \multicolumn{7}{|c|}{ Table 4: Correlation between Histopathological Endometriosis of Individual } \\
\hline
\end{tabular}

\begin{tabular}{|c|c|}
\hline Chi-Square & 4.360 \\
\hline DF & 3 \\
\hline Significance Level & $\mathrm{P}=0.2251$ \\
\hline \multicolumn{2}{|c|}{ Chi-Square Test for Trend } \\
\hline Chi-Square (Trend) & 3.917 \\
\hline DF & 1 \\
\hline Significance Level & $\mathrm{P}=0.0478$ \\
\hline
\end{tabular}

\begin{tabular}{|c|c|c|c|c|c|}
\hline & \multicolumn{4}{|c|}{ Laparoscopic Staging Based on Modified AFS Score } & Total \\
\hline Endometriosis & Stage I & Stage II & Stage III & Stage IV & $\mathbf{1 3}$ \\
\hline Present & $\mathbf{2}$ & $\mathbf{5}$ & $\mathbf{1 2}$ & $\mathbf{3 2}(\mathbf{6 4 \% )}$ \\
\hline Absent & 3 & 6 & 5 & 4 & $18(36 \%)$ \\
\hline \multicolumn{7}{|c|}{ Table 5: Comparison of Laparoscopic Staging with Histopathological Diagnosis of Endometriosis } \\
\hline
\end{tabular}




\begin{tabular}{|c|c|c|c|c|c|c|}
\hline \multirow[b]{2}{*}{$\begin{array}{l}\text { Laparoscopic Stage } \\
\text { (No. of Patient) }\end{array}$} & \multicolumn{5}{|c|}{ No. of Sites Inaccessible to Biopsy } & \multirow[b]{2}{*}{ Total } \\
\hline & $\begin{array}{c}\text { Right } \\
\text { Uterosacral (18) }\end{array}$ & $\begin{array}{c}\text { Left } \\
\text { Uterosacral (17) }\end{array}$ & $\begin{array}{c}\text { Pouch of } \\
\text { Douglas (7) }\end{array}$ & $\begin{array}{l}\text { Right Ovarian } \\
\text { Fossa (9) }\end{array}$ & $\begin{array}{l}\text { Left Ovarian } \\
\text { Fossa (9) }\end{array}$ & \\
\hline Stage $1(5)$ & 0 & 0 & 0 & 0 & 0 & 0 \\
\hline Stage $2(11)$ & 0 & 0 & 0 & 0 & 0 & 0 \\
\hline Stage $3(17)$ & 4 & 4 & 1 & 0 & 1 & 10 \\
\hline Stage 4 (17) & 14 & 13 & 6 & 9 & 8 & 50 \\
\hline
\end{tabular}

\section{DISCUSSION}

In this study, we have determined whether laparoscopy is adequately sensitive to accurately diagnose endometriosis and whether it is adequately specific to replace histopathology in diagnosing endometriosis.

Table 2 shows that laparoscopy has overall sensitivity of about $96 \%$ and NPV of $97 \%$, the sensitivity and NPV of peritoneal endometriosis being about $94.4 \%$ and $96 \%$, but that of laparoscopic visualisation of chocolate cyst has $100 \%$ sensitivity and NPV. On the other hand, visual vs. histological evidence of endometriosis has only about $52.3 \%$ specificity and $44.4 \%$ PPV in case of peritoneal lesion, but about $76.5 \%$ specificity, $83.3 \%$ PPV in case of chocolate cyst. Similar results were found by other authors. ${ }^{[4]}$ But Table 3 shows that the visual evidence of peritoneal endometriosis at a single site has poor sensitivity and NPV (Varies from $54.55 \%$ to $75 \%$ and $63.64 \%$ to $72.22 \%$ depending on the site) for diagnosis of endometriosis in a patient. So to exclude a diagnosis of endometriosis in a patient or to detect all patients with endometriosis, we have to inspect all the peritoneal surfaces for presence or absence of endometriosis. Laparoscopic demonstration of chocolate cyst is highly sensitive as well as specific for diagnosis of ovarian endometriosis.

In our study (Table 1), laparoscopic evidence of endometriosis on uterosacral ligaments has moderate $(0.4<\kappa \geq 0.6)$ agreement with a positive histological diagnosis among the peritoneal sites, while other sites have only fair agreement $(0.2<\kappa \geq 0.4)$, although the number of biopsies are actually less from these sites due to inaccessibility. But it was observed (Table 6) that all of the sites inaccessible to laparoscopic biopsy belong to higher stage patients, specially to stage 4 ( 50 out of 60 ) and about $80 \%$ of stage 4 patients in our study have uterosacral ligaments inaccessible to laparoscopy, lesion at these sites having the highest agreement with a positive histological diagnosis. So diagnosis of endometriosis based on strict histological criteria in higher laparoscopic stage of the disease may lead to erroneous diagnosis due to inadequacy of biopsy specimen. It is also evident from the fact that in our study five patients all having stage 4 disease had no accessible peritoneal site for biopsy, two of which were refuted a histological diagnosis of endometriosis, the rest three being diagnosed on the basis of ovarian endometriosis. The other two stage 4 patients who were refuted histological diagnosis of endometriosis had no evidence of ovarian endometriosis, neither by laparoscopy nor by histopathology and the uterosacral ligaments were inaccessible to laparoscopy.

The presence or absence of histologically proven endometriosis (Table 5) has a linear trend ( $p$ value for trend $=0.0478$ ) with increasing stage of endometriosis, higher the laparoscopic staging of the patient there is more chance of endometriosis to be confirmed in that patient. Strict histological criteria will confirm the surgical diagnosis of endometriosis in approximately $50-65 \%$ of cases only.[3,4]
Similar studies have shown that biopsy specimens even from normal-appearing peritoneum can provide histological confirmation of endometriosis in $0 \%$ to $13 \%$ of cases.[6] So there is a paucity of data on the relevance of histopathologic diagnosis of endometriosis.[7] Histopathologic diagnosis of endometriosis is based on the presence of endometrial-like glands, endometrial stroma and hemosiderin deposit, either within histiocytes or in the stroma. This classic triad may however not be present or may be obscured by haemorrhage, thus resulting in false-negatives. Therefore, negative histology results do not necessarily exclude endometriosis. Actually, in our study four patients ( 2 in stage 3 and 2 in stage 4) with visual diagnosis of chocolate cyst had diagnosis of haemorrhagic cyst on histopathology and one laparoscopically unclassified cyst had the diagnosis of benign ovarian cyst with fibrosis in absence of any discernible epithelial lining on histopathology. Another Four patients had only evidence of inflammation and fibrosis from their available peritoneal biopsy and the one had no peritoneal biopsy due to inaccessibility and hence refuted the diagnosis of endometriosis.

False-positives identified for endometriosis by laparoscopy in this study population included fibrosis, inflammatory changes, normal peritoneum for peritoneal biopsy sample and for adnexal mass serous cysts, haemorrhagic cysts, corpus luteum cysts, normal ovarian stroma, ovarian hyperthecosis, etc.

\section{CONCLUSION}

In this study, we have seen that except in the case of ovarian endometriosis laparoscopy though adequately sensitive to diagnose endometriosis lacks the specificity. So to conclude, histopathology should always be combined with laparoscopy for accurate diagnosis of endometriosis in case of evidence of any suggestive or even doubtful lesion, but is not mandatory in absence of lesion anywhere in pelvis. Laparoscopic staging of endometriosis based on modified AFS score has a positive correlation with histological diagnosis of endometriosis.

\section{REFERENCES}

1. Abstracts from INDO-US workshop on microarray in endometriosis: clinics to research laboratory 3-7 November 2009.

2. Kennedy S, Bergqvist A, Chapron C, et al. ESHRE guideline for the diagnosis and treatment of endometriosis. Hum Reprod 2005;20(10):2698-27704.

3. Stegmann BJ, Sinaii N, Liu S, et al. Using location, colour, size, and depth to characterize and identify endometriosis lesions in a cohort of 133 women. Fertil Steril 2008;89(6):1632-6.

4. Walter AJ, Hentz JG, Magtibay PM, et al. Endometriosis: correlation between histologic and visual findings at laparoscopy. Am J Obstet Gynecol 2001;184(7):1411-3. 
5. Revised American society for reproductive medicine classification of endometriosis: 1996. Fertil Steril 1997;67(5):817-21.

6. Murphy AA, Green WR, Bobbie D, et al. Unsuspected endometriosis documented by scanning electron microscopy in visually normal peritoneum. Fertil Steril 1986;46(3):522-4.
7. Wykes CB, Clark TJ, Khan KS. Accuracy of laparoscopy in the diagnosis of endometriosis: a systematic quantitative review. BJOG 2004;111(11):1204-12. 